\title{
Burnout e sua relação com o clima organizacional em funcionários de um hospital
}

\author{
Burnout and organizational climate relationships in hospital workers
}

Sônia Maria Pessoto dos Santos, Vanessa de Sousa, Fabián Javier Marín Rueda

Programa de Pós-Graduação em Psicologia da Universidade São Francisco (USF) - São Paulo (SP), Brasil.

DOI: http://dx.doi.org/10.7322/abcshs.v40i1.697

\section{RESUMO}

Introdução: Em contextos em que os contatos interpessoais são intensos, os profissionais estão sujeitos a diversas situações de estresse. A síndrome de burnout advém do contato com estressores interpessoais crônicos provenientes do ambiente de trabalho, e seu desenvolvimento é influenciado por alguns aspectos, entre eles, os socioambientais. Objetivo: Entender como se dá a interação entre os sintomas da síndrome de burnout e algumas variáveis do clima organizacional no ambiente hospitalar. Métodos: A amostra foi composta por 565 profissionais de um hospital do interior do estado de São Paulo. A idade variou de 18 a 63 anos, sendo $72,2 \%$ do sexo feminino. Os dados foram coletados no ambiente hospitalar, no período de outubro a dezembro de 2012, respeitando-se os devidos procedimentos éticos. Para a análise dos dados, foram realizadas as estatísticas descritivas, assim como teste $t$ de Student e correlação de Pearson. Resultados: Os resultados mostraram que o fator exaustão emocional do MBI-HSS teve correlações estatisticamente significativas e negativas com todas as dimensões da CLIMOR, sendo elas moderadas com os fatores comunicação, integração e satisfação $(r=-0,45)$; desenvolvimento profissional e benefícios $(r=-0,46)$; e pontuação total $(r=-0,52)$. As correlações dos fatores despersonalização e realização profissional do MBIHSS foram de magnitudes baixas com todas as dimensões da CLIMOR. Conclusão: Os dados corroboram a relação entre o clima organizacional e alguns sintomas do burnout. Há necessidade de que novas pesquisas sejam realizadas com delineamento e análises estatísticas mais sofisticadas e, ainda, que tenham grupos equiparados de pessoas com e sem síndrome de burnout.

Palavras-chave: burnout, clima organizacional; ambiente hospitalar.

\begin{abstract}
Introduction: In contexts in which interpersonal contacts are intense, professionals are subjected to various stress situations. The burnout disorder comes from contact with chronic interpersonal stressors from the work environment and its development is influenced by some aspects, among them, the social and environmental factors. Objective: To understand how is the interaction between the symptoms of burnout and some variables of organizational climate in the hospital setting. Method: The sample consisted of 565 professionals from a hospital in the country region of the state of São Paulo. The age ranged from 18 to 63 , and $72.2 \%$ were female. Data were collected in the hospital setting, from 2012 October to 2012 December, following the ethical procedures. Data analysis consisted in descriptive and $t$-test and Pearson correlation. Results: The results showed that emotional exhaustion factor of the MBI-HSS showed correlations statistically significant and negative with all dimensions of CLIMOR, which were moderate correlations with the factors communication, integration and satisfaction $(r=-0,45)$; professional development and benefits $(r=-0.46)$; and total score $(r=-0.52)$. Correlations of factors depersonalization and personal accomplishment of the MBIHSS were low with all dimensions of the CLIMOR. Conclusion: The data corroborate the relationship between organizational climate and some symptoms of burnout. There is need for further research to be carried out with more sophisticated experimental design and statistical analyzes, and yet, using similar groups of people with and without burnout.
\end{abstract}

Keywords: burnout; organizational climate; hospital setting.

Recebido em: 29/05/2014

Revisado em: 14/10/2014

Aprovado em: 21/10/2014

Autor para correspondência: Fabián Javier Marín Rueda - Universidade São Francisco - Rua Alexandre Rodrigues Barbosa, 45 - CEP: $13251-900$ - Itatiba (SP),

Brasil - E-mail: fabianrueda@saofrancisco.edu.br

Conflito de interesses: nada a declarar. 


\section{INTRODUÇÃO}

Burnout refere-se a uma síndrome psicológica que resulta do contato com estressores interpessoais crônicos provenientes do ambiente de trabalho. Ela é considerada um risco ocupacional em profissões que, predominantemente, exigem contato interpessoal intenso ${ }^{1}$.

Maslach e Jackson ${ }^{2}$ propuseram três dimensões para a síndrome: exaustão emocional, despersonalização e realização profissional. $\mathrm{O}$ aumento da exaustão emocional é o aspecto fundamental da síndrome e acontece quando o sentimento de ser sobrecarregado esgota os recursos emocionais e físicos do indivíduo; já a despersonalização está relacionada a respostas negativas que o indivíduo passa a desenvolver no trabalho e surge como tentativa dele de lidar com tensões emocionais; por fim, na realização profissional, o indivíduo tende a desenvolver sentimentos de incompetência e improdutividade diante da atividade laboral.

A síndrome de burnout pode prejudicar gravemente a qualidade de atendimentos ou serviços prestados por uma equipe. Além disso, pode afetar a vida pessoal do funcionário, ocasionando problemas familiares e, até mesmo, o uso de drogas e álcool ${ }^{2}$.

No ambiente hospitalar, os profissionais estão sujeitos a diversas situações estressantes, pois ficam, frequentemente, próximos aos pacientes, que demandam, muitas vezes, uma atenção constante. Além disso, eles estão em contínuo contato com a dor, o sofrimento e a morte. Nesse contexto, em que os profissionais lidam com necessidades diretas e intensas, pode haver uma maior concentração de sintomas do burnout ${ }^{3}$.

Em relação à incidência de burnout em profissionais da saúde, o estudo de Guido et al. ${ }^{4}$ buscou avaliar o nível de burnout em uma equipe multiprofissional de residentes. Os resultados apontaram que $37,84 \%$ da amostra apresentou alto índice de exaustão emocional, 43,24\% nível alto de despersonalização e 48,65\% baixa realização profissional. Os pesquisadores verificaram que $27 \%$ dos participantes apresentaram os sintomas, que associados caracterizam a síndrome. Outro estudo ${ }^{5}$, dessa vez realizado com enfermeiros, revelou que os participantes apresentaram níveis moderados de exaustão emocional, despersonalização e baixa realização profissional no trabalho.

Quando avaliaram a prevalência de burnout em uma amostra de médicos anestesiologistas, Serralheiro et al. ${ }^{6}$ verificaram que $79,7 \%$ dos anestesistas apresentaram nível de burnout baixo, 16,9\%, nível médio e 3,4\% apresentaram nível alto. Os autores consideram que a presença da síndrome no grupo, ainda que baixa, merece atenção em razão dos danos que seus sintomas provocam.

A síndrome de burnout é influenciada, em seu desenvolvimento, por aspectos socioambientais ${ }^{2}$. Nesse sentido, torna-se relevante a investigação do grau em que algumas dessas características podem estar relacionadas aos sintomas do burnout. Considerando os elementos constituintes do fenômeno, conhecido como clima organizacional, buscou-se, neste estudo, conhecer como se dá a interação entre os sintomas da síndrome de burnout e as variáveis do clima organizacional no ambiente hospitalar.
O clima organizacional pode ser definido como a qualidade do ambiente interno de uma organização e resulta do comportamento e da conduta dos seus membros. Ele serve como base para interpretação de situações, possibilitando a elaboração de intervenções, a fim de alcançar um ambiente favorável para a realização das atividades laborais ${ }^{7}$. Decorre, ainda, do peso dos efeitos de sistemas culturais, valores, políticas, tradições, estilos de lideranças, comportamentos e de como cada colaborador internaliza e reage a esses elementos ${ }^{8}$.

A relação entre a síndrome de burnout e o clima organizacional foi estudada com profissionais inseridos no contexto da saúde por Tomás ${ }^{9}$. O autor pesquisou uma amostra de 236 enfermeiros que cuidavam de toxicodependentes, e foi verificado que a variação dos níveis do burnout acompanha a dos níveis de algumas subescalas do clima organizacional.

Considerando que tanto o clima organizacional quanto a síndrome de burnout podem afetar diretamente as organizações e as relações de trabalho, o presente estudo pretendeu verificar a correlação entre essas variáveis no contexto hospitalar, utilizando, para tanto, o Maslach Burnout Inventory - Human Survey $(M B I-H S S)^{10}$ e a Escala de Avaliação do Clima Organizacional CLIMOR $^{11}$. Esta pesquisa visou contribuir para a compreensão de variáveis que possam promover ou reduzir o risco do burnout em hospitais.

\section{MÉTODOS}

Trata-se de um estudo descritivo e correlacional, pois se configura como um delineamento que busca avaliar o grau de relações entre variáveis ${ }^{12}$. Para a realização da pesquisa, contou-se com uma amostra de 565 profissionais, trabalhadores de um hospital do interior do Estado de São Paulo. A coleta foi efetivada com todos os funcionários da instituição, no período de outubro a dezembro de 2012. A amostra foi composta pelas equipes administrativa, enfermagem, de apoio (responsáveis pelos serviços gerais) e externa (responsáveis pelos atendimentos externos ao hospital), como Serviço de Atendimento Médico de Urgência (SAMU) e Serviço de Atendimento a Pacientes Crônicos (SAEC). A idade dos participantes variou de 18 a 63 anos $(M=31,00 ; D P=9,00)$, sendo $72,2 \%$ do sexo feminino. As informações referentes ao departamento e sexo podem ser observadas na Tabela 1.

Em relação aos procedimentos, primeiramente, foi realizado o contato com a diretoria do hospital para solicitar a autorização das aplicações dos instrumentos. Após o consentimento, o projeto foi submetido ao Comitê de Ética em Pesquisa da Universidade São Francisco, que aprovou sua realização, registrando-a no parecer $\mathrm{n}^{\circ} 76301 / 12$.

Os sujeitos foram abordados em suas unidades de trabalho, onde foram explicados os objetivos da pesquisa, a não obrigatoriedade da participação e a garantia de anonimato. Os funcionários concordaram com a participação no estudo e, então, foram 
direcionados a uma sala de treinamento do hospital, onde assinaram o Termo de Consentimento Livre e Esclarecido e responderam aos instrumentos. Foram chamados 20 colaboradores de cada vez, de diferentes áreas, para não prejudicar a assistência aos pacientes. Os questionários foram respondidos no horário administrativo e nos plantões noturnos pares e ímpares. As aplicações foram realizadas em, aproximadamente, 40 minutos.

Os instrumentos utilizados foram o Maslach Burnout Inventory Human Survey (MBI-HSS) e a Escala de Avaliação do Clima Organizacional - CLIMOR. O MBI-HSS é composto por 22 itens, e o profissional deve atribuir uma nota de um a cinco, relatando a frequência com que as situações propostas foram vivenciadas por ele no seu cotidiano de trabalho. As três dimensões que constituem esse inventário são exaustão emocional, despersonalização e baixa realização pessoal no trabalho. Foi utilizada, neste estudo, a versão traduzida e adaptada por Benevides-Pereira ${ }^{10}$.

A CLIMOR avalia a percepção que os colaboradores têm de sua empresa e do ambiente de trabalho. Ela é composta por 32 itens, respondidos por meio de uma escala Likert de cinco pontos, em que o trabalhador deve se posicionar desde "discordo totalmente" até "concordo plenamente". As dimensões comunicação, integração e satisfação; desenvolvimento profissional e benefícios; ergonomia; condições de trabalho e processo decisório compõem o instrumento.

$\mathrm{Na}$ análise de dados, foram realizadas as estatísticas descritivas de cada fator do MBI-HSS e da CLIMOR. Posteriormente, foram verificadas as correlações entre as dimensões dos instrumentos e, para tanto, utilizou-se a correlação de Pearson, com nível de significância de 0,05 . Além disso, foi realizado o teste $t$ de Student, com o objetivo de verificar se as médias de pontuação dos participantes na CLIMOR diferenciavam-se quando considerados os grupos de pessoas com e sem síndrome de burnout.

\section{RESULTADOS}

Em relação à $M B I-H S S$, na Tabela 2, pode-se observar que a média dos escores dos trabalhadores nas dimensões exaustão emocional, despersonalização e realização profissional foram, respectivamente, 18,88; 8,63 e 31,94. Consideram-se, como grau médio, as pontuações que variam de 17 a 26 na dimensão exaustão emocional; de 7 a 12 na dimensão despersonalização; e de 30 a 38 na realização profissional ${ }^{13}$. Assim, observa-se que os resultados deste estudo indicam graus médios para a amostra geral nas três dimensões do inventário.

A síndrome de burnout caracteriza-se por altos valores de exaustão emocional e despersonalização, e baixos valores de realização profissional ${ }^{2}$. Observou-se que 14 pessoas $(2,48 \%$ da amostra pesquisada) apresentaram sintomas característicos dessa síndrome.

No que se refere à CLIMOR, observou-se que os aspectos relacionados às dimensões comunicação, integração e satisfação; ergonomia; condições de trabalho, assim como a pontuação total, estiveram um pouco abaixo da média. Em outras palavras, a comunicação entre os colaboradores e setores da empresa, as condições físicas do local de trabalho, a remuneração, assim como aspectos globais do ambiente de trabalho, são percebidos pelos trabalhadores como pouco adequados.

A menor média, considerada baixa, foi a da dimensão processo decisório. Com isso, pode-se dizer que a autonomia para tomar decisões relacionadas ao trabalho realizado foi percebida como um aspecto bastante negativo pelos trabalhadores. Já a dimensão desenvolvimento profissional e benefícios apresentou pontuação ligeiramente acima da média da amostra normativa. Esse dado indica que os trabalhadores percebem de uma forma positiva as oportunidades de treinamento e promoções que a empresa oferece.

A seguir, serão apresentadas as correlações de cada uma das dimensões do MBI-HSS com as dimensões e a pontuação total da CLIMOR. Os coeficientes aqui encontrados serão considerados de acordo com a classificação proposta por Dancey e Reidy ${ }^{12}$, a qual sugere que as magnitudes fortes têm coeficientes entre 0,70 e 0,99; as moderadas entre 0,69 e 0,40; e as fracas entre 0,39 e 0,10 .

Pode-se verificar na Tabela 3 que os coeficientes da dimensão exaustão emocional e despersonalização foram negativos, e da realização profissional, positivos. O fator exaustão emocional do MBI-HSS apresentou correlações estatisticamente significativas

Tabela 1: Distribuição de participantes por sexo e departamento

\begin{tabular}{|l|r|r|r|r|r|r|}
\hline & \multicolumn{4}{|c|}{ Sexo } & \multicolumn{3}{c|}{ Total } \\
\hline & \multicolumn{1}{|c|}{ Masculino } & \multicolumn{1}{|c|}{ Feminino } & & \\
\hline & $\mathbf{n}$ & $\%$ & $\mathbf{n}$ & $\%$ & $\mathbf{n}$ & $\%$ \\
\hline Administrativo & 24 & 26,7 & 66 & 73,3 & 90 & 15,9 \\
\hline Enfermagem & 39 & 14,6 & 229 & 85,4 & 268 & 47,4 \\
\hline Apoio & 37 & 26,6 & 102 & 73,4 & 139 & 24,7 \\
\hline Externo & 56 & 82,4 & 12 & 17,6 & 68 & 12,0 \\
\hline Total & 156 & 27,7 & 409 & 72,3 & 565 & 100 \\
\hline
\end{tabular}

Tabela 2: Estatísticas descritivas dos instrumentos

\begin{tabular}{|c|c|c|c|c|}
\hline & Mínimo & Máximo & Média & $\begin{array}{l}\text { Desvio } \\
\text { padrão }\end{array}$ \\
\hline \multicolumn{5}{|l|}{ MBI-HSS } \\
\hline Exaustão emocional & 9 & 44 & 18,88 & 5,84 \\
\hline Despersonalização & 5 & 24 & 8,63 & 2,98 \\
\hline Realização profissional & 8 & 40 & 31,94 & 4,76 \\
\hline \multicolumn{5}{|l|}{ CLIMOR } \\
\hline $\begin{array}{l}\text { Comunicação, integração } \\
\text { e satisfação }\end{array}$ & 13 & 55 & 39,64 & 8,22 \\
\hline $\begin{array}{l}\text { Desenvolvimento } \\
\text { profissional e benefícios }\end{array}$ & 9 & 40 & 27,15 & 6,50 \\
\hline Ergonomia & 6 & 30 & 18,81 & 5,00 \\
\hline Condições de trabalho & 4 & 20 & 10,31 & 3,26 \\
\hline Processo decisório & 3 & 15 & 7,89 & 3,19 \\
\hline CLIMOR total & 52 & 150 & 103,79 & 18,66 \\
\hline
\end{tabular}

MBI-HSS: Maslach Burnout Inventory - Human Survey 
Tabela 3: Matriz de correlação entre os fatores e pontuação total da CLIMOR e as Dimensões do Maslach Burnout Inventory Human Survey

\begin{tabular}{|l|c|c|c|}
\hline $\begin{array}{l}\text { Exaustão } \\
\text { emocional }\end{array}$ & $\begin{array}{c}\text { Desperso- } \\
\text { nalização }\end{array}$ & $\begin{array}{c}\text { Realização } \\
\text { pessoal }\end{array}$ \\
\hline $\begin{array}{l}\text { Comunicação, } \\
\text { integração e } \\
\text { satisfação }\end{array}$ & $-0,45^{\star}$ & $-0,24^{\star}$ & $0,23^{\star}$ \\
$\begin{array}{l}\text { Desenvolvimento } \\
\text { profissional e } \\
\text { benefícios }\end{array}$ & $-0,46^{\star}$ & $-0,17^{\star}$ & $0,23^{\star}$ \\
\hline $\begin{array}{l}\text { Ergonomia } \\
\text { Condições de }\end{array}$ & $-0,36^{\star}$ & $-0,12^{\star}$ & $0,13^{\star}$ \\
\hline trabalho & $-0,21^{\star}$ & $-0,02$ & 0,04 \\
\hline $\begin{array}{l}\text { Processo } \\
\text { decisório }\end{array}$ & $-0,14^{*}$ & $-0,04$ & 0,04 \\
\hline $\begin{array}{l}\text { CLIMOR- } \\
\text { pontuação total }\end{array}$ & $-0,52^{\star}$ & $-0,21^{\star}$ & $0,23^{\star}$ \\
\hline
\end{tabular}

${ }^{*} p<0,01$

e negativas com a pontuação total e com todas as dimensões da CLIMOR. Os coeficientes foram moderados com comunicação, integração e satisfação; desenvolvimento profissional e benefícios e com a pontuação total. Já as correlações foram baixas (de $-0,14$ a -0,36) com as dimensões ergonomia, condições de trabalho e processo decisório.

Em relação ao fator despersonalização do $M B I-H S S$, pode-se observar que houve correlação significativa com três das cinco dimensões da CLIMOR e também com a pontuação total. Todos os índices foram negativos e de magnitude baixa. Entre eles, a correlação com a dimensão comunicação, integração e satisfação foi a maior $(r=-0,24)$. Assim, verificou-se que a despersonalização esteve pouco associada à avaliação de oportunidades e possibilidades de promoção na empresa $(r=-0,17)$. Os coeficientes foram menores em relação à ergonomia $(\mathrm{r}=-0,12)$ e não foram significativos com condições de trabalho e poder decisório.

Já no que concerne ao fator realização profissional do $M B I-$ $H S S$, verificou-se que as correlações foram positivas com todas as pontuações da CLIMOR. Os índices foram estatisticamente significativos em quatro das seis correlações possíveis. As magnitudes foram todas baixas e, dentre elas, as de maiores índices foram com os fatores comunicação, integração e satisfação; desenvolvimento profissional e benefícios e pontuação total $(r=0,23 ; r=0,23$ e $r=0,23$, respectivamente).

Com o objetivo de verificar se a presença ou ausência da síndrome de burnout diferencia significativamente os participantes em relação às médias na pontuação da CLIMOR, foi realizado o teste $t$ de Student. Foi verificado que a média da pontuação total da CLIMOR foi significativamente maior no grupo que não apresentou a síndrome $(\mathrm{t}=-3,757, \mathrm{p}<0,001)$. No entanto, esse resultado deve ser visto com ressalvas, pois os dois grupos em questão foram constituídos com números de participantes bastante distintos, sendo que o grupo de pessoas que sofre de burnout $(\mathrm{M}=85,50 ; \mathrm{DP}=18,49)$ foi composto por 14 participantes, enquanto o grupo que não sofre da síndrome $(\mathrm{M}=104,25 ; \mathrm{DP}=16,06)$ teve 551 indivíduos.

\section{DISCUSSÃO}

A sociedade capitalista, legitimando os comportamentos competitivos e acirrando a busca pela inserção no mercado de trabalho, traz para o cotidiano dos profissionais de diversas áreas exigências contínuas de produção. Tais aspectos fazem com que os trabalhadores estejam cada vez mais vinculados ao ambiente laboral.

O trabalho, no contexto hospitalar, expõe o trabalhador ao contato com o sofrimento, tanto de pacientes quanto dos familiares, a riscos ocupacionais e, não raro, à sobrecarga de trabalho ${ }^{2}$. Informações relevantes sobre a prevalência da síndrome de burnout em profissionais da saúde têm sido apontadas por pesquisadores $^{4-6,9}$.

Há um crescente interesse de estudiosos e psicólogos organizacionais em conhecer a síndrome de burnout e o clima organizacional, no entanto há poucos estudos sobre a relação dessas variáveis. Assim, esses construtos foram pesquisados no contexto hospitalar com o objetivo de conhecer o grau em que estão mutuamente relacionados, o que pode contribuir para a compreensão de variáveis que promovem ou reduzem o risco do burnout em hospitais.

Os resultados do presente estudo apontaram que a dimensão do MBI-HSS que está mais associada ao clima organizacional é a exaustão emocional. Vale ressaltar que a exaustão emocional é tida como a dimensão mais representativa da síndrome ${ }^{2}$, e que é comum que amostras tenham maiores médias nesse fator, conforme mostram os estudos aqui citados.

Os fatores da CLIMOR que tiveram maior associação com essa dimensão foram comunicação, integração e satisfação; desenvolvimento profissional e benefícios, além da pontuação total. O fator comunicação, integração e satisfação refere-se aos canais de comunicação da organização, à cooperação e ao relacionamento entre os setores e funcionários, e ao sentimento dos funcionários em relação à organização. A correlação da exaustão emocional com esse fator sugere que são menos suscetíveis à exaustão emocional os colaboradores que utilizam os canais de comunicação para se relacionar bem com os colegas e com outras equipes. Esse dado vai ao encontro das concepções de Leiter e Maslach ${ }^{14,15}$, que afirmam que o contato com colegas de trabalho e a percepção de uma rede de apoio entre eles pode ajudar a desacelerar o processo de estabelecimento do burnout.

Outros autores também afirmam que a falta de apoio social está intimamente ligada à síndrome ${ }^{1}$. Além disso, apontam a falha na comunicação, ou falta de feedback, como um aspecto frequentemente ligado ao burnout. Tomás ${ }^{9}$ observou uma correlação moderada entre envolvimento e exaustão emocional, além de ter verificado que o aumento da segunda é provocado, em parte, por diminuições nos escores da primeira dimensão aqui citada.

A correlação entre exaustão emocional e desenvolvimento profissional e benefícios, observada neste estudo, indica que as pessoas que estão esgotadas emocionalmente são também aquelas que não estão satisfeitas com os benefícios e com os 
treinamentos que proporcionam desenvolvimento de carreira ou possível promoção. Esse resultado corrobora a concepção de Maslach, Schaufeli e Leiter ${ }^{1}$ que afirmam que a falta de possibilidade para a ascensão na instituição é uma variável ligada aos sintomas do burnout. Esses autores esclarecem ainda que, quando as expectativas de desenvolvimento profissional são consideradas irrealistas ou idealistas, essa atitude pode ser um fator de risco para o burnout.

Monteiro et al. ${ }^{16}$ contam que a falta de reconhecimento, que pode ser refletida pela falta de benefícios e oportunidades de desenvolvimento de carreira, foi relatada por trabalhadores de um hospital em uma pesquisa sobre o adoecimento psíquico. Os indivíduos mencionaram sentimentos de menos valia e desmotivação como sendo características associadas aos seus sofrimentos psíquicos.

No que diz respeito à correlação da exaustão emocional e pontuação geral da CLIMOR, é possível inferir que, de modo geral, quanto melhor a percepção global que os colaboradores têm do ambiente de trabalho menos susceptíveis eles são a se sentirem exaustos emocionalmente e, assim, menos propensos ao burnout.
A relação entre a exaustão emocional e os demais fatores da CLIMOR - ergonomia, condições de trabalho e processo decisório - foi baixa. Os aspectos físicos de uma organização, a remuneração, bem como a autonomia dos funcionários, foram apontados, na literatura, como variáveis que influenciam o estabelecimento da síndrome ${ }^{1}$. No entanto, nesta amostra, essas relações apresentaram-se baixas. Percebe-se que os fatores mais ligados ao convívio e reconhecimento social tiveram mais relação com exaustão que os demais. É interessante notar que Maslach et al..$^{15}$ afirmaram recentemente que uma estratégia de intervenção bastante válida no contexto organizacional é a mudança na qualidade do ambiente de trabalho, enfatizando justamente as ligações e o envolvimento interpessoal.

Este trabalho apontou algumas variáveis do clima organizacional que estão relacionadas ao burnout no ambiente hospitalar. Certamente, há necessidade de aprofundamento dos resultados obtidos. Sugere-se, assim, a realização de estudos longitudinais, com delineamento e análises estatísticas mais sofisticadas. Além disso, seria interessante observar se esses resultados se manteriam em uma amostra maior de pessoas com a presença da síndrome de burnout; já que a quantidade restrita dessa amostra foi uma limitação do presente estudo.

\section{REFERÊNCIAS}

1. Maslach C, Schaufeli WB, Leiter MP. Job burnout. Annu Rev Psychol. 2001;52:397-22.

http://dx.doi.org/10.1146/annurev.psych.52.1.397

2. Maslach C, Jackson SE. The measurement of experienced burnout. J Occupat Behavior. 1981;2(2):99-13. http://dx.doi.org/10.1002/job.4030020205

3. Benevides-Pereira AMT. As atividades de enfermagem em hospital: um fator de vulnerabilidade ao Burnout. In: BenevidesPereira AMT. Burnout: quando o trabalho ameaça o bem-estar do trabalhador. São Paulo: Casa do Psicólogo; 2002.

4. Guido LA, Silva RM, Goulart CT, Bolzan MEO, Lopes LFD. Síndrome de burnout em residentes multiprofissionais de uma universidade pública. Rev Esc Enferm USP. 2012;46(6):1477-83. http://dx.doi.org/10.1590/S0080-62342012000600027

5. Rissardo MP, Gasparino RC. Exaustão emocional em enfermeiros de um hospital público. Esc Anna Nery. 2013;17(1):128-32. http://dx.doi.org/10.1590/S1414-81452013000100018

6. Serralheiro FC, Braga ALF, Garcia MLB, Grigio T, Martins LC. Prevalência da síndrome de Burnout em anestesiologistas de Instituição de Ensino Superior em Medicina. Arq Bras Ciênc Saúde. 2011;36(3):140-3. http://dx.doi.org/10.7322/abcs.v36i3.52

7. Abbey A, Dickson JW. R\&D work climate and innovation in semiconductors. Acad Manag J. 1983;26(2):362-8. http://dx.doi.org/10.2307/255984

8. Edela LPS. Clima e Cultura Organizacionais como se manifestam e como se manejam. Porto Alegre: Edgar Blucher; 1978.
9. Tomás CC. Riscos Psicossociais e Clima Organizacional: Burnout nos enfermeiros que cuidam de toxicodependentes. Rev Toxicodependências. 2011;17(2):23-30.

10. Benevides-Pereira AMT. MBI - Maslach Burnout Inventory e suas adaptações para o Brasil. In: Anais da $32^{a}$ Reunião Anual de Psicologia. Rio de Janeiro, 2001. p.84-85.

11. Rueda FJM, Santos AAA. Escala de Avaliação do Clima Organizacional (CLIMOR). São Paulo: Vetor; 2011.

12. Dancey CP, Reidy J. Estatística sem matemática para psicologia. Porto Alegre: Artmed; 2006.

13. Vasques-Menezes I. A contribuição da psicologia clínica na compreensão do burnout: um estudo com professores. Tese (Doutorado em Psicologia) - Instituto de Psicologia da Universidade de Brasília, Brasília, 2005.

14. Leiter MP, Maslach C. The impact of interpersonal environment on burnout and organizational commitment. J Organizat Behavior. 1988;9(4):297-308.

http://dx.doi.org/10.1002/job.4030090402

15. Maslach C, Leiter MP, Jackson SE. Making a significant difference with burnout interventions: researcher and practitioner collaboration. J Organizat Behavior. 2012;33(2):296-300. http://dx.doi.org/10.1002/job.784

16. Monteiro JK, Oliveira ALL, Ribeiro CS, Grisa GH, Agostini N. Adoecimento Psíquico de Trabalhadores de Unidades de Terapia Intensiva. Psicol Ciênc Prof. 2013;33(2):366-79. http://dx.doi.org/10.1590/S1414-98932013000200009 